\title{
Sequence based typing of HLA-A and B Exons-2 and -3 in a HIV-positive native community with limited HLA diversity from the North of Argentina
}

\author{
DA Dilernia', D Mónaco ${ }^{1 *}$, R Coloccini ${ }^{1}$, M Pando ${ }^{1}$, M Quipildor $^{2}$, A Di Paolo², E Hunter ${ }^{3}$, H Salomon ${ }^{1}$ \\ From AIDS Vaccine 2012 \\ Boston, MA, USA. 9-12 September 2012
}

\section{Background}

We previously reported a limited diversity of HLA-class I alleles in two-digits typing studies of HIV-positive native populations from Oran, North of Argentina. In the present study we determine whether the restricted diversity observed at low-resolution reflects also a restricted genetic diversity in HLA peptide groove.

\section{Methods}

We studied 65 HIV-positive patients whose HLA-A and -B genes were previously typed by SSOP technique. We setup a sequence-based typing of the most prevalent alleles in Oran. HLA-A and B were initially PCR-amplified and exons-2 and -3 were sequenced. NCBI-SBT-Interpretation tool was used to confirm the two-digits typing with previous SSOP data. We designed a set of primers specifics for HLAs highly prevalent in Oran to achieve differential PCR-amplification of each allele in heterozygote patients. Phylogenetic analysis was used to assign exons-2 and -3 sequences to a high-resolution HLA group.

\section{Results}

Our results show that for HLA-A alleles, $85.1 \%$ of $\mathrm{A}^{*} 02$ are $A * 02: 01: 01: 01,96.7 \%$ of $A * 31$ are $A * 31: 01: 02$ and $92.8 \%$ of $A * 24$ are $A * 24: 02: 01: 01$. In the case of $A * 68,50 \%$ are $A * 68: 01: 02$ and $31.2 \%$ are $A * 68: 17$. For HLA-B alleles, $B * 35$ was diverse: $B * 35: 01: 01: 01$ (15.8\%), $B * 35: 04: 01$ (15.8\%), B*35:05:01 (21.1\%) and B*35:19 (21.1\%). 43.8\% of $B * 39$-alleles were $B * 39: 05: 01$ and $25 \%$ were $B * 39: 03$. 52.9\% of $B * 48$-alleles were $B * 48: 01: 01$ and $35.3 \%$ were $B^{*} 48: 03: 01$. $62.5 \%$ of $B * 51$ alleles were $B * 51: 01: 01$. The mentioned alleles represent the $73.1 \%$ of HLA-A genetic diversity and the $45.4 \%$ of HLA-B. All the polimorphisms observed lead to non-synonymous changes.

\section{Conclusion}

Our results show that two-digits typing of HLA-A usually corresponds with a specific allele in our population. For HLA-B alleles, observed within-subtype diversity was higher. The different protein sequence encoded by exons2 and -3 may lead to different peptide specificities among alleles from the same HLA-B subtype that would be missclassified as homogeneous in a low-resolution typing study.

\section{Author details}

${ }^{1}$ Argentinean Reference Center for AIDS, Buenos Aires, Argentina. ${ }^{2}$ Hospital San Vicente de Paul, San Ramón de la Nueva Oran, Argentina. ${ }^{3}$ Emory Vaccine Center, Atlanta, GA, USA.

Published: 13 September 2012

doi:10.1186/1742-4690-9-S2-P164

Cite this article as: Dilernia et al.: Sequence based typing of HLA-A and B Exons-2 and -3 in a HIV-positive native community with limited HLA diversity from the North of Argentina. Retrovirology 2012 9(Suppl 2):P164.

${ }^{1}$ Argentinean Reference Center for AIDS, Buenos Aires, Argentina

Full list of author information is available at the end of the article

(c) 2012 Dilernia et al; licensee BioMed Central Ltd. This is an Open Access article distributed under the terms of the Creative Commons Attribution License (http://creativecommons.org/licenses/by/2.0), which permits unrestricted use, distribution, and reproduction in any medium, provided the original work is properly cited. 